Recherches en didactique des langues et des cultures

Les cahiers de l'Acedle

16-1 | 2019

Enseigner la phonétique d'une langue étrangère

\title{
Théorie, recherche et didactique de la prosodie et de l'intonation en L2 : nouvelles perspectives
}

Fabián Santiago

\section{OpenEdition}

Journals

Édition électronique

URL : http://journals.openedition.org/rdlc/4545

DOI : $10.4000 /$ rdlc.4545

ISSN : 1958-5772

Éditeur

ACEDLE

Référence électronique

Fabián Santiago, «Théorie, recherche et didactique de la prosodie et de l'intonation en L2 : nouvelles perspectives », Recherches en didactique des langues et des cultures [En ligne], 16-1 | 2019, mis en ligne le 30 janvier 2019, consulté le 19 avril 2019. URL : http://journals.openedition.org/rdlc/4545; DOI : $10.4000 /$ rdlc. 4545

Ce document a été généré automatiquement le 19 avril 2019

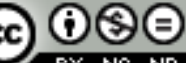

Recherches en didactique des langues et des cultures is licensed under a Creative Commons AttributionNonCommercial-NoDerivatives 4.0 International License 


\title{
Théorie, recherche et didactique de la prosodie et de l'intonation en L2 : nouvelles perspectives
}

\author{
Fabián Santiago
}

\section{Introduction ${ }^{1}$}

1 La prosodie fait partie des compétences orales que tout apprenant doit développer pour mieux communiquer dans une L2. Elle véhicule plusieurs sens linguistiques et accomplit des fonctions discursives. Les éléments prosodiques, i.e. l'intonation, l'accentuation et le rythme, se traduisent par la réalisation de montées ou de descentes mélodiques de la voix, la réalisation de syllabes accentuées, la présence de pauses ou l'allongement temporel des noyaux vocaliques. Bien que ces éléments prosodiques soient essentiels dans l'intelligibilité du discours en L2 (Billières, 2008 ; Chun, 2002 ; Munro \& Derwin, 1995), les études sur leur acquisition et leur enseignement en français L2 ont été largement délaissées. Cette contribution essaie de renouer le lien entre les recherches actuelles en prosodie du français L1 et son acquisition en tant que L2. Nous défendons la nécessité d'intégrer les nouvelles connaissances de ces recherches (en tant que phénomène linguistique) dans les aspects pédagogiques, le tout pour essayer d'établir un nouveau pont entre les recherches scientifiques actuelles et les pratiques didactiques en L2.

\section{Les modèles prosodiques utilisés dans la didactique du français $L 2$}

Les ouvrages destinés à l'enseignement du français L2 (Léon, 1976 ; Lauret, 2007 ; Detey et al., 2016) ont toujours souligné deux aspects du système prosodique de cette langue. D'une part, le français est décrit comme une langue ayant un accent de phrase dont la principale fonction est de délimiter les frontières droites des groupes prosodiques. 
D'autre part, les contours mélodiques prototypiques employés dans cette langue se réduisent généralement à deux : montants et descendants. Une analyse plus fine de ces ouvrages nous montre que les règles sous-jacentes à l'émergence de la structure accentuelle ainsi que le nombre d'intonèmes (contours mélodiques associés à une fonction linguistique) ne font pas toujours consensus. En plus, dans la plupart de ces descriptions, le statut phonologique des phénomènes prosodiques n'est pas clairement défini, d'autant moins les contextes discursifs dans lesquels ces formes prosodiques émergent. Dans les sections qui suivent, nous aborderons sommairement quelques aspects que ces descriptions pourraient intégrer afin de rendre ces références plus robustes, plus contextualisées et plus proches des nouvelles connaissances que nous avons du système prosodique du français.

\section{La structure accentuelle et le phrasé prosodique en français L2}

3 Le français, langue sans accent lexical, possède un système accentuel caractérisé par la présence d'un accent dit final (appelé aussi accent de groupe ou accent de phrase) dont la fonction principale est de découper le matériel linguistique de l'énoncé (fonction démarcative) afin de créer des groupes accentuels (GA) appelés aussi rythmiques (Di Cristo, 2016). L'accent participe à la construction du phrasé prosodique en français : le flux de parole est structuré ou découpé en groupes prosodiques qui peuvent varier de taille accomplissant différentes fonctions dans la hiérarchie prosodique de la langue. Les ouvrages didactiques abordent cette question sans faire allusion explicitement aux contraintes qui déterminent la formation des GA. Par exemple, Léon (1976:130) propose que « l'accent tonique est toujours placé sur la dernière voyelle prononcée ». Ainsi, les dernières syllabes des mots Paris, administration, et immensité devraient être réalisées avec un accent final. Mais lorsque ces mots entrent dans un groupe, ils perdent leur accent au profit de celui-ci : Paris est une ville, l'administration française, etc. Pour cette auteure, un GA représente une idée et accomplit une fonction grammaticale. Pour sa part, Lauret (2007) ajoute que des informations sémantico-syntaxiques sont nécessaires dans la distribution des accents. Ainsi, bien que les deux exemples ci-dessous soient formés avec les mêmes segments (consonnes et voyelles), la place des accents (et leur nombre) diffère en fonction du sens des énoncés.

[Jean] [porte] [le sac] = ['zã.'pobt.lə.'sak].

[J'emporte] [le sac] = [3ã.'pobt.lə.'sak].

4 Le même auteur reconnaît que si un groupe nominal ayant la fonction de sujet de la phrase n'a qu'une seule syllabe, le prédicat doit être découpé en plusieurs GA. Ainsi, le découpage en (3) serait peu naturel (indiqué par l'astérisque), alors que celui en (4) serait le plus fréquent en français :

*[Paul] [est sorti de son bureau discrètement].

[Paul] [est sorti] [de son bureau] [discrètement].

Ces quelques exemples nous montrent certaines inconsistances. D'une part, ces descriptions ne précisent pas quels types de mots sont potentiellement accentuables, à savoir : les mots lexicaux. D'autre part, l'opacité des concepts idée et la clarté des informations sémantico-syntaxiques nécessaires pour la structuration de la parole ne permettent pas d'identifier les contraintes que les apprenants doivent considérer pour 
segmenter les énoncés. Ainsi, les élèves et les enseignants ont le droit de se demander pourquoi le découpage en (6) ne serait pas plus approprié que celui proposé par Léon (1976: 158) en (5), alors que le découpage en (7) serait pratiquement impossible.

[Je serai au Champs-Elysées] [à deux heures cet après-midi]. [Je serai] [au Champs-Elysées] [à deux heures] [cet après-midi]. *[Je serai au] [Champs-Elysées à] [deux] [heures cet après-midi].

Une description appropriée à l'enseignement du français se revendiquant comme plus contextualisée et adaptée à l'apprenant du français L2 doit répondre à plusieurs questions qui restent peu claires dans la plupart de ces exemples : Quelles sont les contraintes qui permettent de construire les GA ? Quel est le rôle des GA dans les interactions orales ? Dans quelle mesure le contexte discursif détermine la production des GA des énoncés ? Quelles sont les caractéristiques phonétiques associées à la production des GA qu'il faut produire ? Il est reconnu aujourd'hui que plusieurs contraintes interviennent dans l'émergence des GA qui rendent compte du phrasé prosodique ${ }^{2}$. Ces contraintes sont modulées via l'interaction des informations syntaxiques, morpho-phonologiques, rythmiques et sémantico-pragmatiques. Les contraintes qui ont été largement étudiées et qui sont généralement négligées dans les ouvrages didactiques sont les suivantes :

- Contraintes syntaxiques. Un groupe accentuel (GA) est formé par tout mot lexical (noms, verbes, adjectifs...) avec les clitiques (déterminants, prépositions...) qui en dépendent à sa gauche. La fonction de l'accent final est de marquer la frontière droite de ce GA (Post, 2000 ; Martin, 2009). L'exemple en (6) illustre l'application de cette contrainte ou chaque mot lexical forme avec ses clitiques (je, au, à et cet) un GA qui est borné par un accent final à sa droite.

- Contraintes phonologiques. Un mot potentiellement porteur d'un accent final peut être désaccentué afin d'éviter une collision accentuelle comme dans l'exemple (8a), où un seul GA est formé à la place de deux (Post, 2000), ou dans l'exemple (6) où un réajustement permet le regroupement de deux mots lexicaux dans un seul GA (à deux heures). L'autre possibilité est le recul d'accent d'une syllabe pour le premier accent, comme l'exemple donnée en (8b) tiré de Martin (2009).

- Contraintes rythmiques. Les GA ont tendance à contenir un même nombre de syllabes (Martin, 2009 ; Wioland, 2005). L'exemple en (9) montre les endroits où les accents peuvent être réalisés selon les contraintes syntaxiques ci-dessus. A droite de la flèche, un réajustement est observé afin de créer deux GA de même taille (4 syllabes chacun). De plus, en moyenne, les GA contiennent entre trois et sept syllabes (les GA contenant moins de 2 syllabes ou plus de 7 syllabes sont rares).

- Contraintes sémantiques et pragmatiques (fonction discursive). La place de l'accent peut indiquer un contraste de sens dans certains cas. En (10) un accent réalisé sur le mot jaune avec une montée mélodique sert à clarifier une information (focus contrastif) : je ne veux pas un pull vert, mais jaune (Di Cristo, 2016).

a. [Une maison] [jaune] >> [Une maison jaune]

b. [Le chocolat] [chaud] >> [le chocolat] [chaud]

[Marie] [adore] [les chocolats] >> [Marie adore] [les chocolats]

[Je veux un pull jaune] (sans focus) vs [Je veux un pull JAUNE] (avec focus)

L'intégration de ces contraintes dans la didactique du français L2 clarifie le fait que la formation des GA dépend de l'interaction de plusieurs types d'informations et évite une version réductrice et rigide de la construction du phrasé en français. En particulier, la 
contrainte syntaxique y gagne en clarté pour les apprenants. Même si dans la production orale des natifs la structure prosodique ne suit pas fidèlement les contraintes syntaxiques (Delais-Roussarie et al., 2011), les apprenants peuvent s'appuyer sur la syntaxe dans un premier temps pour mieux comprendre la formation de la structure prosodique en français, car cette contrainte ne dit pas où un accent doit être réalisé, mais plutôt où il peut être produit. Il leur est ensuite possible d'intégrer les autres règles de réajustement avec l'expérience et l'enseignement explicite/implicite. Ces contraintes d'ordre formel sont d'une grande abstraction pour les apprenants. Il serait donc souhaitable de ne pas négliger la place des contraintes sémantiques/pragmatiques, car à la différence du phonéticien ou du linguiste, un apprenant a surtout intérêt à connaître la fonction du phrasé prosodique dans les interactions orales de tous les jours. Les contraintes sémantiques et pragmatiques viennent accomplir de manière plus parlante ce besoin : l'apprenant doit être conscient que, dans certains cas, le phrasé prosodique et la place de l'accent peut aider à mieux interpréter la sémantique des énoncés.

Enfin, le dernier élément concerne la réalisation phonétique des GA. Les ouvrages pédagogiques proposent généralement que l'accent final des GA est produit avec des mouvements mélodiques montants, mais ils ignorent souvent que cette montée mélodique doit être accompagnée d'un allongement temporel suffisant de la voyelle (Di Cristo, 2016). En outre, si le contour montant est prototypique en français, il n'est pas rare de trouver des accents finaux réalisés avec des contours mélodiques descendants (Martin, 1987) ${ }^{3}$. Enfin, la représentation des caractéristiques phonétiques est souvent donnée dans les manuels avec l'emploi de flèches ou pseudo-pentagrammes pour simuler la modulation de la voix. Cependant, nous croyons qu'une représentation phonétique plus pédagogique consisterait à visualiser le tracé de f $0^{4}$, son alignement avec les syllabes ainsi que les allongements temporels. Ceci permettrait à l'apprenant d'intégrer les informations acoustiques, perceptives et visuelles (abstraites) en même temps (Herment, 2018). La figure suivante illustre l'oscillogramme (onde sonore), le tracé de fo (courbe noire) représentant les mouvements mélodiques de la phrase en (5) avec le logiciel Praat (Boersma \& Weenink, 2018). Les flèches bleues indiquent la direction du mouvement mélodique associé à la fin de chaque GA ainsi que son alignement avec le noyau syllabique, et les flèches rouges l'allongement temporel associé aux syllabes accentuées. Dans cet exemple, il est intéressant de noter que l'accent final du premier GA est produit par un contour descendant, et non montant (comme il souvent décrit dans les manuels). 
Figure 1 : Visualisation des GA et du tracé de f0 d'un énoncé en français



\section{La production et la perception des contours mélodiques prototypiques}

9 L'enseignement de l'intonation d'une L2 nécessite le choix d'un modèle théorique. En anglais L2, les contours mélodiques sont souvent décrits en termes de configurations nucléaires selon l'école britannique (O'Connor \& Arnold, 1973; Wells 2006; Herment 2018). En espagnol L2, ils sont décrits en termes de niveaux de hauteur tonale et de cadence/anti-cadence suivant les travaux de Quilis (1993). En français L2, les ouvrages didactiques, comme celui de Lauret (2007), prennent quasiment tous comme base les travaux de Delattre (1966) où les contours mélodiques sont décrits également en termes de forme (hauteur tonale et direction du mouvement mélodique), oppositions (assertion vs question, continuation vs question, etc.) et les possibles commutations. Toutefois, il n'y a pas un accord général sur le nombre et le type de contours intonatifs prototypiques qu'il faut enseigner en français L2, ni sur leur fonction dans le système : ChampagneMuzar \& Bourdages (1998) proposent qu'entre 2 et 4 contours sont nécessaires dans l'apprentissage du français L2, Léon (1976) en propose deux, alors que Detey et al. (2016) n'en font aucune mention particulière.

Ces divergences sont déjà observées dans les modèles théoriques actuels de l'intonation en français L1, et ce, pour plusieurs raisons. Tout d'abord, l'identification des mouvements mélodiques linguistiquement pertinents dans le système n'est pas une tâche facile, car il y a plusieurs critères à considérer : ils peuvent véhiculer un contraste de sens au niveau de l'énoncé (assertion vs question), ils émergent dans le signal de parole selon les règles de distribution de la langue (certaines formes mélodiques apparaissent dans certaines positions dans l'énoncé), et ils accomplissent une fonction discursive et/ou dialogique (ils expriment un doute, une certitude, une demande d'information). Or, les modèles décrivant l'intonation du français ne donnent pas la même priorité à ces critères. Le deuxième problème est le manque de descriptions robustes qui expliquent clairement la façon dont ces contours mélodiques sont communément réalisés dans la langue cible à partir des observations dans les corpora de parole spontanée. Le troisième problème est que l'association forme-fonction (fonction discursive) en matière d'intonation n'est pas 
biunivoque, mais bien au contraire, une forme intonative peut être associée à différentes fonctions discursives dans le système. Delattre (1966) avait proposé 10 contours mélodiques ayant une fonction linguistique en français. Mais, d'après les recherches actuelles, il est admis que ce modèle n'est plus complètement valide aujourd'hui. Par exemple, le contour de continuation majeure (mouvement mélodique indiquant que le locuteur a l'intention de continuer son discours) et le contour montant d'une question totale neutre (qui exige la réponse oui/non sans marquage pragmatique particulier) ont les mêmes traits sur la hauteur mélodique dans ce modèle : les deux contours commencent dans la zone bas du registre tonal du locuteur et atteignent la zone aigue (voir dans la figure 2 les niveaux 2 et 4 respectivement). Ces deux contours s'opposeraient par leur forme : convexe pour la continuation majeure et concave pour la question.

Figure 2 : Stylisation de deux types de contours montants chez Delattre (1966)

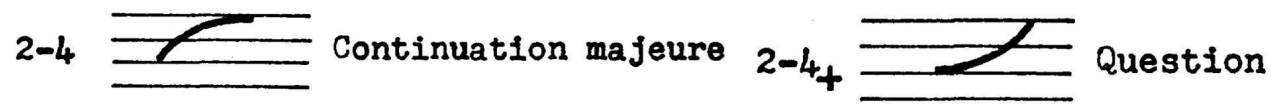

11 Les recherches actuelles ont montré que la forme du contour (convexe vs concave) ne peut pas véhiculer un tel contraste : le contour d'une question et celui d'une continuation dite majeure sont généralement produits et perçus de manière similaire chez les locuteurs francophones natifs (Post, 2000 ; Di Cristo, 2016). De manière similaire, différents auteurs ont déjà montré que d'autres contours proposés par Delattre n'ont pas un statut phonologique, comme c'est le cas de la continuation mineure, le commandement et l'exclamation (Martin, 2009 ; Di Cristo, 2016).

12 Tout ceci nous amène à penser qu'une grammaire de référence en L2 basée sur les travaux de Delattre (1966) est discutable aujourd'hui. Au contraire, nous considérons qu'une grammaire de référence en L2 serait plus appropriée aux apprenants si elle explique clairement les critères qui déterminent le statut phonologique des contours. Vient ensuite la question de la fonction de tels contours : elle se situe au niveau purement phonologique (classification des contours en termes de leur forme et distribution dans les énoncés) et/ou discursif (rôle discursif de ces contours dans les interactions orales). Enfin, cette grammaire de référence doit décrire, sur la base des recherches menées en parole spontanée et en perception, la forme prototypique dudit contour. Sur ce point, il semblerait que les recherches adoptant un cadre formaliste soient les mieux avancées (Di Cristo, 2016 ; Delais-Roussarie et al., 2015 ; Post, 2000). Viennent ensuite les travaux sur les rapports entre intonation et fonctions discursives dans un cadre interactionniste et dialogique (i.e., les rapports entre intonation et actes de parole comme ceux étudiés par Portes et al., 2014). Sous une approche formelle, nous pouvons constater que les auteurs s'accordent sur le statut d'au moins quatre schémas intonatifs prototypiques en français qui seraient observés à la fin des groupes intonatifs $(\mathrm{GI})^{5}$, à savoir : montant, descendant, montant-descendant depuis la syllabe pré-tonique et montant-descendant dans la dernière syllabe. Ces quatre contours mélodiques ont été attestés dans de larges corpus oraux et testés au niveau perceptif auprès de locuteurs natifs au niveau plutôt phonologique. Nous essayons de résumer les propriétés de ces contours dans le tableau 1. 
Tableau 1 : Les quatre contours prototypiques destinés à l'enseignement du français $L 2$ : forme, distribution et fonction

\begin{tabular}{|c|c|c|c|c|}
\hline \multirow[t]{2}{*}{ Contour } & \multirow[t]{2}{*}{ Descendant } & \multirow[t]{2}{*}{ Montant } & \multicolumn{2}{|l|}{ Montant-descendant } \\
\hline & & & Version 1 & Version 2 \\
\hline \multicolumn{5}{|l|}{ Stylisation } \\
\hline Description & $\begin{array}{lr}\text { Le contour de } \\
\text { f0 descend à } \\
\text { partir ru } \\
\text { début de } & \text { la } \\
\text { dernière } & \\
\text { syllabe du } & \text { GI } \\
\text { jusqu'à ra la } \\
\text { zone basse du } \\
\text { registre du } \\
\text { locuteur. }\end{array}$ & $\begin{array}{l}\text { Le contour de fo } \\
\text { monte à partir } \\
\text { du début de la } \\
\text { dernière syllabe } \\
\text { du GI. }\end{array}$ & $\begin{array}{l}\text { Le contour de f0 } \\
\text { commence à } \\
\text { monter depuis la } \\
\text { pénultième syllabe } \\
\text { et atteint un pic } \\
\text { dans celle-ci, } \\
\text { ensuite, il descend } \\
\text { à partir de la } \\
\text { dernière syllabe du } \\
\text { GI jusqu'à la fin de } \\
\text { l'énoncé ou jusqu'à } \\
\text { la zone médiane } \\
\text { du locuteur. }\end{array}$ & $\begin{array}{l}\text { Le contour de f0 } \\
\text { commence à monter } \\
\text { depuis la } \\
\text { pénultième syllabe } \\
\text { du GI et atteint un } \\
\text { pic au début de la } \\
\text { dernière syllabe } \\
\text { nucléaire puis, il } \\
\text { descend } \\
\text { généralement } \\
\text { jusqu'à à la zone } \\
\text { médiane du registre } \\
\text { tonal du locuteur. }\end{array}$ \\
\hline Distribution & $\begin{array}{l}\text { Groupe } \\
\text { intonatif } \\
\text { terminal. }\end{array}$ & $\begin{array}{l}\text { Groupe intonatif } \\
\text { terminal et non } \\
\text { terminal. }\end{array}$ & $\begin{array}{l}\text { Groupe intonatif } \\
\text { terminal. }\end{array}$ & $\begin{array}{l}\text { Groupe intonatif } \\
\text { terminal (et/ou fin } \\
\text { de groupe accentuel } \\
\text { en position médiane } \\
\text { dans l'énoncé). }\end{array}$ \\
\hline \multirow{2}{*}{$\begin{array}{l}\text { Fonctions } \\
\text { grammaticales } \\
\text { et discursives } \\
\text { (contexte) }\end{array}$} & $\begin{array}{l}\text { - Assertion } \\
\text { - Conclusion } \\
\text { - Ordre }\end{array}$ & $\begin{array}{l}\text { - Questions } \\
\text { totales neutres } \\
\text { sans } \\
\text { marquage } \\
\text { syntaxique } \\
\text { - Continuation } \\
\text { - Enumération }\end{array}$ & \multirow{2}{*}{\multicolumn{2}{|c|}{$\begin{array}{l}\text { Evidence, exclamation, doute, } \\
\text { implication, conviction }\end{array}$}} \\
\hline & \multicolumn{2}{|c|}{$\begin{array}{l}\text { Questions partielles neutres et } \\
\text { questions totales neutres avec } \\
\text { marquage syntaxique }\end{array}$} & & \\
\hline
\end{tabular}

13 L'entrée stylisation représente la forme du contour mélodique à la fin des GI (et/ou GA), en l'occurrence, le tracé de fo observé dans la dernière et l'avant dernière syllabe de ces groupes. La partie description explique la réalisation phonétique de ces contours en termes d'alignement du tracé de fo avec les syllabes. Il est important de noter que ces descriptions prennent comme point d'ancrage (endroit où le contour est réalisé) la syllabe. L'entrée distribution dans ce tableau obéit à des critères formels : distribution des formes mélodiques dans le GI selon la place qu'ils occupent dans le discours : terminal (comme c'est le cas de la fin d'une phrase racine ou à la fin d'un énoncé complet) ou non terminal (à l'intérieur d'une même phrase ou d'un même énoncé). Dans le critère des fonctions discursives, nous récapitulons le sens que ces contours peuvent véhiculer en termes sémantiques et pragmatiques (contexte discursif) selon Portes \& Lancia (2017), Di Cristo (2016) et Martin (2009) : il s'agit ici d'une notion proche de celle d'acte de parole. Enfin, les fonctions dans les cellules grises se réfèrent aux fonctions qui sont encore polémiques et discutables aujourd'hui.

Cette proposition s'avère un peu réductrice pour ce qui est des fonctions discursives (grammaire contextualisée) et des critères syntaxiques dans la dernière ligne, car il s'agit de contraintes plus complexes. Par exemple, les schémas mélodiques des questions totales neutres qui demandent la réponse oui/non (i.e., tu viens ?) sont produites avec différents contours mélodiques selon leur structure syntaxique. Ainsi, les questions construites contenant l'expression est-ce que ou ayant une inversion du pronom sujet (i.e., est-il venu ?) sont produites avec un contour descendant ou avec un contour montant (Di Cristo, 2016). Les questions partielles neutres (construites avec un mot interrogatif du type qui, comment, où...) sont dans le même cas. Delattre (1966) proposait que le contour final dans ces deux types de questions était descendant, tout comme les assertions. Or, il 
est bien connu que le contour dans ces types de questions peut aussi être montant, sans qu'il y ait une préférence apparente. Il est intéressant de noter que la montée finale dans ce type de questions n'a pas généralement la même ampleur que celle d'une question totale : elle est plus discrète (Di Cristo 2016). La figure 3 illustre la variation intonative finale que l'on peut trouver pour ces types de questions.

Figure 3 : Stylisation des deux types de contours terminaux associés aux questions totales avec marquage syntaxique (a) et les questions partielles (b)

(a)

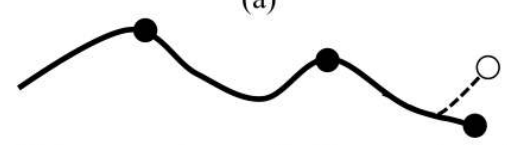

Est-ce que tu as acheté ce cadeau ? (b)

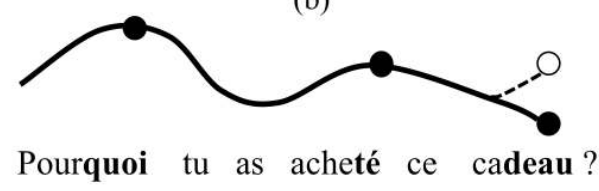

Les questions peuvent avoir des profils mélodiques différents selon qu'elles sont des demandes d'information ou des demandes de confirmation (actes de parole). A l'heure actuelle, les recherches n'ont pas clarifié dans quelle mesure le choix d'un contour montant/descendant est associé à une valeur pragmatique (i.e., l'expression de la politesse $)^{6}$. En conséquence, la fonction discursive pour ces deux cas partage les deux types de contours dans le tableau, lequel donne pour le moment une certaine souplesse à ces descriptions afin d'éviter la rigidité des grammaires traditionnelles. Nous devons noter que ce problème reste quand-même crucial pour les didacticiens/apprenants d'une L2, car l'enseignement des actes de parole est une pratique commune. Comme il y a très peu de recherches empiriques qui clarifient complètement la question, il serait plus judicieux de ne pas faire une association directe entre forme-sens, ou forme-acte de parole. Ce tableau, bien qu'imparfait, essaie de clarifier les différents critères que l'on peut considérer pour la construction d'une nouvelle grammaire de référence pour le français L2 et tente surtout de distinguer les différents critères linguistiques qui sont parfois confondus et mélangés dans plusieurs manuels pédagogiques.

\section{La prosodie du français L2 : acquisition et perspectives didactiques}

16 Si les descriptions grammaticales gagnent en clarté lorsqu'elles ont une certaine souplesse, les enseignants doivent savoir, avant de les enseigner en salle de classe, quelles sont les erreurs prototypiques des apprenants. Les études sur corpus d'apprenants examinant l'intonation en L2 s'avèrent donc indispensables pour comprendre quelles sont les difficultés de l'apprentissage de la prosodie, d'autant que ce type de données peut éclairer certaines inconsistances dans les modèles théoriques en L1 et les grammaires descriptives.

Les études examinant l'acquisition du phrasé prosodique et ses rapports avec la structure accentuelle ne sont pas, malheureusement, très nombreuses en français L2. Quelques études récentes ont montré qu'en règle générale, les étudiants hispanophones arrivent à produire les accents en position oxytonne en français pour marquer les GA (Santiago \& Delais-Roussarie 2015; Schwab 2012 ; Barquero, 2012). Cependant, plusieurs différences sont observées entre la parole des apprenants et celle des natifs. Barquero (2012) \& Schwab (2014) montrent que les hispanophones tendent à produire davantage de GA que 
les francophones natifs dans les assertions : les étudiants ont tendance à accentuer tous les mots lexicaux comme dans leur L1 (i.e., une sur-généralisation de la contrainte syntaxique). Santiago \& Delais-Roussarie (2015) trouvent l'effet contraire dans les questions : les apprenants produisent moins de GA que les natifs. Cette désaccentuation des mots serait motivée par un transfert de la L1, car en espagnol, les mots lexicaux en position interne dans les questions ne sont généralement pas accentués. Santiago (2016) montre aussi que les hispanophones, qu'ils soient intermédiaires ou avancés, ne rallongent pas temporellement les syllabes accentuées comme le font les francophones au moment de produire les GA. De plus, à l'instar des natifs, les apprenants ne produisent pas de contours mélodiques descendants pour marquer les GA.

18 Ces études montrent que la production des accents finaux des GA semble être acquise de manière relativement facile. En revanche, deux types de problèmes sont observés : (i) les étudiants semblent ignorer les contraintes rythmiques et phonologiques dans la construction des GA et en produisent trop/moins, (ii) les implémentations phonétiques ne sont pas similaires à celles des natifs (l'allongement temporel associé à l'accent final ne semble pas être produit comme chez les natifs, les contours descendants ne sont pas observés, etc.).

19 Pour ce qui est de la structure intonative, Gabriel et al. (2018) montrent que les apprenants germanophones sur-emploient les contours montants à la fin des questions partielles. En d'autres termes, l'emploi des contours descendants dans ce type de questions est rare chez les apprenants, alors que ces contours sont plus fréquents chez les natifs. Cette observation avait déjà été faite par Santiago \& Delais-Roussarie (2015) pour le cas des apprenants hispanophones. Particulièrement, ces derniers auteurs rapportent que l'ampleur du contour montant dans ce type de questions diffère beaucoup de ce qu'on observe chez les natifs : chez les apprenants hispanophones débutants, par exemple, la montée finale atteint fréquemment la zone aigue du registre tonal (contour extramontant), un contour qui n'est pas observé ni chez les natifs francophones, ni chez les natifs hispanophones.

L'analyse de toutes ces recherches permettrait au didacticien de faire des prédictions plus robustes sur les erreurs prosodiques prototypiques des apprenants et de les situer dans un premier temps au niveau phonologique ou au niveau phonétique. Par exemple, la production d'une question partielle avec un contour (extra-)montant final en français L2 n'entraîne pas un changement de sens de l'énoncé, donc la sur-généralisation de l'emploi de ce contour ne relèverait pas du domaine phonologique (i.e., les représentations catégorielles de la langue cible sont appropriées). En revanche, ces déviances prosodiques seraient plutôt d'ordre phonétique, tout particulièrement, la production d'un contour extra-haut n'est pas fréquente en français L1. Cette analyse peut changer en fonction de la L1 des apprenants et de leur niveau, d'où l'importance d'analyser ces erreurs en profondeur. Par exemple, les locuteurs sinophones n'emploient pas nécessairement les contours intonatifs pour indiquer la modalité interrogative des énoncés dans leur L1. Il serait normal que chez ces locuteurs l'acquisition des contours montants associés aux questions relève du niveau phonologique et phonétique, car chez eux, la représentation catégorielle du contour montant associé aux questions est inexistante.

21 Pour finir, il ne serait pas étonnant que les apprenants ayant comme L1 une langue romane commencent à produire tous les types de questions neutres avec des contours mélodiques montants. Le didacticien peut enseigner aux étudiants que, dans le cas des questions où la modalité est marquée syntaxiquement, le contour montant n'est pas 
obligatoire, et si le contour montant est utilisé, celui-ci a une ampleur plus modérée que pour celles des questions totales.

Les recherches en acquisition sont sans doute indispensables pour pouvoir identifier les problèmes qu'affrontent les étudiants de français L2. Ces résultats ont un double objectif : (i) comprendre l'interlangue des apprenants et son évolution dans l'apprentissage, (ii) confronter les productions natives et non natives pour éclairer certains aspects des modèles théoriques qui restent en suspens. En revanche, pour identifier les erreurs prosodiques (qu'elles soient au niveau phonologique ou phonétique), les didacticiens doivent compter sur une grammaire de la prosodie et de l'intonation qui explicite clairement les différentes interfaces qui existent avec les autres domaines linguistiques. Si les études dans la production sont le mieux représentées, il ne faut pas négliger le besoin d'explorer les aspects perceptifs en L1 et en L2. En effet, étudier seulement la production des apprenants ne suffit pas pour identifier quels sont les aspects faciles/ difficiles à acquérir. Il convient d'insister aussi sur le fait que les aspects perceptifs sont indispensables pour pouvoir mesurer les effets de la prosodie non native dans les interactions orales. Pour le moment, nous ne savons pas à quel point la prosodie non native rapportée ici a des effets sur l'intelligibilité des messages chez les locuteurs natifs. Tous ces résultats seraient sans doute éclairants pour identifier l'importance de ces erreurs.

\section{Quelles perspectives dans la recherche en didactique de L2?}

L'étape la plus intéressante pour les lecteurs didacticiens concerne sans doute les aspects pédagogiques: Quels aspects prosodiques enseigner ? À quel moment ? Pourquoi ? Avec quelles techniques ? La littérature consacrée à la didactique de la prosodie et de la prononciation en général est abondante en anglais L2, et les études empiriques qui ont mesuré les effets positifs dans l'enseignement de la prosodie avec des approches théoriques plus récentes ont donné des résultats très encourageants (Estevas-Vilaplana, 2016). Les études en français L2 évaluant différents types de techniques pédagogiques sont encore rares, comme celle d'Alazard (2013) qui a montré que les effets de la gestuelle corporelle sont très positifs pour développer la fluence en $\mathrm{L} 2$, y compris le phrasé prosodique. Nous considérons que les recherches en didactique pourraient avancer sur trois aspects : (i) la proposition des grammaires de référence mieux adaptées aux descriptions modernes et empiriques, (ii) l'analyse de l'acquisition de la prosodie en français L2 avec des données d'apprenants venant de plusieurs L1 afin de relever les erreurs prototypiques, (iii) l'adaptation des contenus prosodiques à enseigner en fonction du niveau de l'apprenant. Ce n'est qu'avec ces résultats que les didacticiens pourraient concevoir des techniques pédagogiques spécifiques plus robustes et appropriées pour améliorer la compétence prosodique des apprenants. 


\section{BIBLIOGRAPHIE}

Alazard, Ch. (2013). Rôle de la prosodie dans la fluence en lecture oralisée chez des apprenants de Français Langue Etrangère. Thèse de doctorat. Université de Toulouse 2.

Barquero, M. A. (2012). "A Comparative study on Accentual Structure between Spanish Learners of French Interlanguage and French Native Speakers". In Ma, Q., Ding, H. \& Hirst, D. (dir.), Proceedings of the 6th International Conference on Speech Prosody. Shanghai: Tongji University Press, pp. 250-253.

Billières, M. (2008). « Le statut de l'intonation dans l'évolution de l'enseignement/apprentissage de l'oral en FLE ». Le Français dans le Monde, Recherches et applications, vol. 43, pp. 27-37.

Boersma, P. \& Weenink, D. (2018). Praat. http://www.fon.hum.uva.nl/praat/

Champagne-Muzard, C. \& Bougardes, J. (1998). Le point sur la phonétique. Paris : CLE International.

Chun, D. M. (2002). Discourse Intonation in L2: From Theory and Research to Practice.

Amsterdam/Philadelphia: John Benjamins Publishing Company.

Delais-Roussarie, E. et al. (2015). "Intonational phonology of French : Developing a ToBI system for French". In S. Frota, \& P. Prieto, (dir.). Intonation in Romance. Oxford: Oxford University Press, pp. 63-100.

Delais-Roussarie, E., Yoo, H. \& Post, B. (2011). « Quand frontières prosodiques et frontières syntaxiques se rencontrent ». Langue française, $\mathrm{n}^{\circ} 170$, pp. 29-44.

Delattre, P. (1966). «Les dix intonations de base du français ». The French Review, vol. 40, no. 1, pp. 1-14.

Detey, S., Racine, I., Kawaguchi, Y. \& Eychenne, J. (2016). La prononciation du français dans le monde. Paris : CLE International.

Di Cristo, D. (2016). Les musiques du français parlé. Berlin/Boston : Walter de Gruyter.

Estevas-Vilaplana, E. (2016). "The Teaching and Learning of L2 English Intonation in a Distance Education Environment: TL_TOBI vs. the Traditional Models". Linguistica, vol. 57, no. 1, pp. 73-91. Gabriel, C., Meisenburg, T. \& Wocker, B. (2018). "Intonation and (Re)syllabification in L2 French Interrogatives Produced by L1 German Learners: Comparing Different Proficiency Levels". Proceedings of the 9th International Conference on Speech Prosody. Poznań, Poland, pp. 833-837.

Herment, S. (2018). « Apprentissage et enseignement de la prosodie : l'importance de la visualisation ». Revue française de linguistique appliquée, vol. 23, no. 1, pp. 73-88.

Jenkins, J. (2004). "Research in Teaching Pronunciation and Intonation". Annual Review of Applied Linguistics, vol. 24, pp. 109-125.

Lauret, B. (2007). Enseigner la prononciation du français : questions et outils. Paris : Hachette.

Léon, M. (1976). Exercices systématiques de prononciation française. Paris : Hachette/Larousse.

Martin, Ph. (2009). L'intonation du français. Paris : Armand Colin. Collection U.

Martin, Ph. (1987). "Prosodic and Rhythmic Structures in French." Linguistics, vol. 25, no. 5, pp. 925-949. 
Michelas, A., Portes, C. \& Champagne-Lavau M. (2015). "When Pitch Accents Encode Speaker Commitment: Evidence from French Intonation". Language and Speech, vol. 59, pp. 266-293.

Munro, M. J. \& Derwing, T. M. (1995). "Foreign Accent, Comprehensibility, and Intelligibility in the Speech of Second Language Learners". Language Learning, vol. 45, pp. 73-97.

O'Connor, J. D. \& Arnold, G. F. (1973). Intonation of Colloquial English. London: Longman.

Post, B. (2000). Tonal andPphrasal Structures in French Intonation, Hague: Holland Academic Graphics.

Portes, C. \& Lancia, L. (2017). "Earlier or Higher ? Comparing French Rising-Falling Contour with Rising Contour in a Corpus of Conversation". Journal of phonetics, vol. 63, pp. 35-52.

Portes, C., Beyssade, C., Michelas, A., Marandin, J.M. \& Champagne-Lavau, M. (2014). "The Dialogical Dimension of Intonational Meaning : Evidence from French". Journal of Pragmatics. vol. 74 , pp. 15-29.

Quilis, A. (1993). Tratado de fonología y fonética españolas. Madrid: Gredos.

Santiago, F. (2016). « La prosodie des syntagmes intonatifs en français L2 : une étude perceptive ». Langages, vol. 202, no. 2, pp. 13-33.

Santiago, F. \& Delais-Roussarie, E. (2015). "The Acquisition of Question Intonation by Mexican Spanish Learners of French". In Delais-Roussarie, E., Avanzi, M. \& Herment, S. (dir.). Prosody and Language in Contact: L2 Acquisition, Attrition and Languages in Multilingual Situations. Heldelberg: Springer, pp. 243-270.

Schwab, S. (2012). "Do Native Spanish Speakers Transfer Accentual Acoustic Properties from Spanish to French L2?". In Ma, Q., Ding, H. \& Hirst, D. (dir.). Proceedings of the 6th International Conference on Speech Prosody, Shanghai, vol. 1, Shanghai: Tongji University Press, pp. 330-333.

Wells, J. (2006). English Intonation: An Introduction. Cambridge: Cambridge University Press.

Wioland, F. (2005). Prononcer les mots du français. Paris : Hachette.

\section{NOTES}

1. Je tiens à remercier vivement Cristel Portes pour la lecture attentive de cet article et ses recommandations.

2. Faute de place, nous écartons de cette analyse le rôle de l'accent secondaire ou initial et le rôle de l'accent primaire dans la topicalisation ou le marquage du focus contrastif.

3. Faute de place, nous ne discutons pas ici si les contours descendants en position non finale qui sont obligatoires dans la théorie de Martin (1987).

4. Unité qui désigne le nombre de cycles périodiques de l'onde acoustique décomptés en un seconde et qui correspond au nombre de vibrations des plis vocaux par seconde.

5. Un groupe intonatif (appelé aussi phrase intonative ou groupe de souffle) est une unité prosodique plus large que les GA dont la caractéristique principale est d'être bornée par une flexion mélodique importante laquelle peut entraîner un contraste de sens au niveau de l'énoncé.

6. Voir l'étude de Michelas et al. (2015) qui ont exploré cette question. 


\section{RÉSUMÉS}

Nous discutons de l'importance d'intégrer les résultats des recherches récentes en matière de prosodie et d'intonation dans l'élaboration des descriptions de référence actuelles et contextualisées destinées à l'enseignement du français L2. Nous nous focalisons sur la nature du groupe accentuel, ses rapports avec le phrasé prosodique et le rôle des contours mélodiques. Nous confrontons cette analyse avec les résultats des recherches consacrées à l'acquisition de la prosodie du français en tant que L2. Notre objectif est de renouer les liens entre la recherche en phonologie expérimentale et la didactique de l'intonation du français.

We discuss the results of recent research on experimental phonology and their integration into modern and contextualized grammars for students of L2 French. This paper focuses on the basic properties of Accentual Phrases in French, their relations with prosodic phrasing, and the description of melodic contours. We illustrate how such notions are being investigated within the field of second language acquisition. Our goal is to stress the importance of integrating new findings of experimental phonology into teaching practices of French as an L2.

\section{INDEX}

Mots-clés : prosodie non native, intonation en langue étrangère, didactique de la prosodie du français langue étrangère

Keywords : non-native prosody, second language intonation, teaching L2 French prosody

\section{AUTEUR}

\section{FABIÁN SANTIAGO}

Université Paris 8 \& CNRS (SFL, LPP \& LLF)

Fabian Santiago est maître de conférences (Associate Professor) en Didactique, Acquisition des L2 et Phonétique à l'Université de Paris 8 Vincennes - Saint-Denis. Il est associé au laboratoire Structures Formelles du Langage (UMR 7023 CRNS) où il mène ses recherches sur l'acquisition de la prononciation en L2 et l'intonation du français L2. Ses travaux prennent comme cadre la théorie métrique et auto-segmentale. Récemment il a exploré les rapports du développement de la structure sonore en L2 et les autres niveaux linguistiques en examinant des données riches issues de différents corpora en L2.

fabian.santiago-vargas[at]univ-paris8.fr 